International Journal of Pure and Applied Mathematical Sciences.

ISSN 0972-9828 Volume 10, Number 2 (2017), pp. 177-183

(C) Research India Publications

https://dx.doi.org/10.37622/IJPAMS/10.2.2017.177-183

\title{
Total Resolving Number of Block Graphs and Line Graphs
}

\author{
J. Paulraj Joseph and N. Shunmugapriya \\ Department of Mathematics, \\ Manonmaniam Sundaranar University, \\ Tirunelveli - 627 012, Tamil Nadu, India.
}

\begin{abstract}
Let $G=(V, E)$ be a simple connected graph. An ordered subset $W$ of $V$ is said to be a resolving set of $G$ if every vertex is uniquely determined by its vector of distances to the vertices in $W$. The minimum cardinality of a resolving set is called the resolving number of $G$ and is denoted by $r(G)$. As an extension, the total resolving number was introduced in [6] as the minimum cardinality taken over all resolving sets in which $\langle W\rangle$ has no isolates and it is denoted by $\operatorname{tr}(G)$. In this paper, we obtain the bounds on the total resolving number of block graphs and line graphs. Also, we characterize the extremal graphs.
\end{abstract}

AMS subject classification: Primary 05C12, Secondary 05C35.

Keywords: resolving number, total resolving number, block graph, line graph.

\section{Introduction}

Let $G=(V, E)$ be a finite, simple, connected and undirected graph. The degree of a vertex $v$ in a graph $G$ is the number of edges incident to $v$ and it is denoted by $d(v)$. The maximum degree in a graph $G$ is denoted by $\Delta(G)$ and the minimum degree is denoted by $\delta(G)$. The distance $d(u, v)$ between two vertices $u$ and $v$ in $G$ is the length of a shortest $u-v$ path in $G$. The maximum value of distance between vertices of $G$ is called its diameter. $P_{n}$ denote the path on $\mathrm{n}$ vertices. $C_{n}$ denote the cycle on $n$ vertices. $K_{n}$ denote the complete graph on $n$ vertices. A graph is acyclic if it has no cycles. A tree is a connected acyclic graph. A star is denoted by $K_{1, n-1}$. A tree obtained by joining the centres of two stars $K_{1, s}$ and $K_{1, t}$ by an edge is called a bistar and it is denoted by $B_{s, t}$. The join $G+H$ consists of $G \cup H$ and all edges joining a vertex of $G$ and a vertex of $H$. 
For a cut vertex $v$ of a connected graph $G$, suppose that the disconnected graph $G \backslash\{v\}$ has $k$ components $G_{1}, G_{2}, \ldots, G_{k}(k \geq 2)$. The induced subgraphs $B_{i}=G\left[V\left(G_{i}\right) \cup\{v\}\right]$ are connected and referred to as the brances of $G$ at $v$. A cut vertex $v$ is a path support if there is a nontrivial path as a branch at $v$; a simple path support if there is exactly one path support at $v$; a multi path support if there are more than one path support at $v$. A graph contains exactly one cycle is called a unicyclic graph. A block of a graph $G$ is a maximal connected subgraph of $G$ that has no cut vertex. A block of $G$ containing exactly one cut vertex of $G$ is an end block of $G$. $G$ is called a triangle free graph if it has no triangle. To identify non adjacent vertices $x$ and $y$ of a graph $G$ is to replace these vertices by a single vertex incident to all the edges which were incident in $G$ to either $x$ or $y$.

If $W=\left\{w_{1}, w_{2}, \ldots, w_{k}\right\} \subseteq V(G)$ is an ordered set, then the ordered k-tuple $\left(d\left(v, w_{1}\right), d\left(v, w_{2}\right), \ldots, d\left(v, w_{k}\right)\right)$ is called the representation of $v$ with respect to $W$ and it is denoted by $r(v \mid W)$. Since the representation for each $w_{i} \in W$ contains exactly one 0 in the $i^{t h}$ position, all the vertices of $W$ have distinct representations. $W$ is called a resolving set for $G$ if all the vertices of $V \backslash W$ also have distinct representations. The minimum cardinality of a resolving set is called the resolving number of $G$ and it is denoted by $r(G)$. In [6] we introduced and studied total resolving number. If $W$ is a resolving set and the induced subgraph $\langle W\rangle$ has no isolates, then $W$ is called a total resolving set of $G$. The minimum cardinality taken over all total resolving sets of $G$ is called the total resolving number of $G$ and is denoted by $\operatorname{tr}(G)$.

In this paper, we obtain the bounds on the total resolving number of block graphs and line graphs. Also, we characterize the extremal graphs.

\section{Total Resolving Number of Graphs}

The following results are used in subsequent sections.

Theorem 2.1. [6] For $n \geq 3, \operatorname{tr}\left(P_{n}\right)=2$ and $\operatorname{tr}\left(C_{n}\right)=2$.

Observation 2.2. [6] For any graph $G$ of order $n \geq 3,2 \leq \operatorname{tr}(G) \leq n-1$.

Observation 2.3. [6] Let $G$ be a unicyclic graph with odd cycle $C_{k}$. Then $\operatorname{tr}(G)=2$ if and only if no vertex of $G$ is a multi path support and either at most three consecutive vertices of $C_{k}$ are simple path supports or exactly two non adjacent vertices of $C_{k}$ at a distance two are simple path supports.

Observation 2.4. [6] For any tree $T, \operatorname{tr}(T)=2$ if and only if $T$ is a path.

Theorem 2.5. [6] Let $G$ be a graph of order $n \geq 3$. Then $\operatorname{tr}(G)=n-1$ if and only if $G \cong K_{n}$ or $K_{1, n-1}$.

Remark 2.6. [6] If $G$ has multi-path support, then $\operatorname{tr}(G) \neq 2$.

Observation 2.7. If $\operatorname{tr}(G)=2$, then $G$ contains at most one block of order at least 3 . 
Notation 2.8. [7] If $H$ and $K$ are two graphs, then the graph obtained by identifying one center of $H$ with one center of $K$ is denoted by $H * K$ and the graph obtained by joining one center of $H$ to one center of $K$ is denoted by $H e K$.

Theorem 2.9. [7] Let $G$ be a 1-connected graph of order $n \geq 4$. Then $\operatorname{tr}(G)=n-2$ if and only if $G$ is isomorphic to $B_{s, t}, s \geq 1, t \geq 1$ or $2 K_{3}+e$ or $K_{1, s} * K_{t}, s \geq 1, t \geq 3$ or $K_{3} * K_{t}, t \geq 3$ or $K_{3}$ e $K_{1, s}, s \geq 3$.

\section{Block Graphs}

In this section, we obtain the bounds for the total resolving number of the block graph of a general graph and characterize the extremal graphs.

Definition 3.1. The block graph $B(G)$ of a graph $G$ is a graph whose vertices are the blocks of $G$ and two of these vertices are adjacent whenever the corresponding blocks contain a common cut vertex of $G$.

Observation 3.2. Let $G$ be a graph with $n^{\prime} \geq 3$ blocks. Then $2 \leq \operatorname{tr}(B(G)) \leq n^{\prime}-1$.

Proof. Clearly, the order of $B(G)$ is $n^{\prime}$. By Observation 2.2, $2 \leq \operatorname{tr}(B(G)) \leq n^{\prime}-1$.

Theorem 3.3. [4] A graph $G$ is a block graph of some graph $H$ if and only if the blocks of $G$ are complete.

Definition 3.4. A graph $G$ is said to be a block path if $B(G)$ is a path.

Theorem 3.5. Let $G$ be a graph with $n^{\prime} \geq 3$ blocks. Then $\operatorname{tr}(B(G))=2$ if and only if $G$ satisfies one of the following conditions.

(i) $G$ is a block path

(ii) $G$ has either a unique cut vertex lying in exactly three blocks or a cut vertex $v$ lying in exactly three blocks, all other cut vertices other than $v$ lying in exactly two blocks and every block contains at most two cut vertices.

Proof. Assume that $\operatorname{tr}(B(G))=2$. If $B(G)$ is an acyclic graph, then by Observation $2.4, B(G) \cong P_{n^{\prime}}$. Thus $G$ is a block path. Now, we assume that $B(G)$ contains a cycle. Then by Remark 2.6, Observation 2.7 and Theorem 3.3, clearly, $B(G)$ is a unicyclic graph with a cycle $C_{3}$. By Observation $2.3, G$ has either a unique cut vertex lying in three blocks or a cut vertex $v$ lying in three blocks, all other cut vertices other than $v$ lie in exactly two blocks and every block contains at most two cut vertices.

Conversely, if $G$ satisfies $(i)$, then $B(G) \cong P_{n^{\prime}}$ and hence by Theorem 2.1, $\operatorname{tr}(B(G))=$ 2. If $G$ satisfies $(i i)$, then $B(G)$ is one of the graph given in Observation 2.3 with cycle $C_{3}$ and hence $\operatorname{tr}(B(G))=2$. 
Theorem 3.6. Let $G$ be a graph with $n^{\prime} \geq 3$ blocks. Then $\operatorname{tr}(B(G))=n^{\prime}-1$ if and only if either $G$ has exactly one cut vertex or $G$ has at least two cut vertices and all of them lie in one block such that each one is common to exactly two blocks of $G$.

Proof. Assume that $\operatorname{tr}(B(G))=n^{\prime}-1$. By Theorem $2.5, B(G) \cong K_{n^{\prime}}$ or $K_{1, n^{\prime}-1}$. If $B(G) \cong K_{n^{\prime}}$, then each block of $G$ is an end block. If $B(G) \cong K_{1, n^{\prime}-1}$, then $G$ has one non end block and each cut vertex lying in exactly two blocks. Thus either $G$ has exactly one cut vertex or $G$ has at least two cut vertices and all of them lie in one block such that each one is common to exactly two blocks of $G$.

Conversely, if $G$ has exactly one cut vertex, then $B(G) \cong K_{n^{\prime}}$ and hence by Theorem $2.5, \operatorname{tr}(B(G))=n^{\prime}-1$. If $G$ has at least two cut vertices and all of them lie in one block such that each one is common to exactly two blocks of $G$, then $B(G) \cong K_{1, n^{\prime}-1}$ and hence by Theorem $2.5, \operatorname{tr}(B(G))=n^{\prime}-1$.

Theorem 3.7. Let $G$ be a graph with $n^{\prime} \geq 3$ blocks. Then $\operatorname{tr}(B(G))=n^{\prime}-2$ if and only if $G$ satisfies one of the following conditions.

(i) $G$ has exactly two cut vertices of which one is common to exactly three blocks and another one is common to at least three blocks.

(ii) $G$ has at least two cut vertices and all of them lie in one block such that one is common to at least 3 block each other is common to exactly two blocks of $G$.

(iii) $G$ has exactly three cut vertices of which one is not a vertex of an end block of $G$, other two are in distinct blocks and each one is common to exactly three blocks of $G$.

(iv) $G$ has $t \geq 4$ cut vertices such that $t-1$ vertices are in one block of which each one is common to exactly two blocks of $G$ and the remaining vertex belongs to another block which is common to exactly three blocks.

(v) $G$ has exactly two non end blocks of which one has at least two cut vertices and another one has at least three cut vertices such that each one is common to exactly two blocks of $G$.

Proof. Assume that $\operatorname{tr}(B(G))=n^{\prime}-2$. Using Theorems 2.9 and $3.3, B(G) \cong B_{s, t}$, $s \geq 1$ and $t \geq 2$ or $2 K_{3}+e$ or $K_{1, s} * K_{t}, s \geq 1$ and $t \geq 3$ or $K_{3} * K_{t}, t \geq 3$ or $K_{3} e K_{1, s}$, $s \geq 3$ given in Theorem 2.9. If $B(G) \cong B_{s, t}, s \geq 1$ and $t \geq 2$, then $G$ is the graph given in $(v)$. If $B(G) \cong 2 K_{3}+e$, then $G$ is the graph given in (iii). If $B(G) \cong K_{1, s} * K_{t}$, $s \geq 1$ and $t \geq 3$, then $G$ is the graph given in (ii). If $B(G) \cong K_{3} * K_{t}, t \geq 3$, then $G$ is the graph given in $(i)$. If $B(G) \cong K_{3} e K_{1, s}, s \geq 3$, then $G$ is the graph given in (iv).

Conversely, if $G$ is the graph given in $(i)$, then $B(G) \cong K_{3} * K_{t}$ and hence by Theorem $2.9, \operatorname{tr}(B(G))=n^{\prime}-2$. If $G$ is the graph given in $(i i)$, then $B(G) \cong K_{1, s} * K_{t}, s \geq 1$ and $t \geq 3$ and hence by Theorem 2.9, $\operatorname{tr}(B(G))=n^{\prime}-2$. If $G$ is the graph given in (iii), then $B(G) \cong 2 K_{3}+e$ and hence by Theorem $2.9, \operatorname{tr}(B(G))=n^{\prime}-2$. If $G$ is the graph given in $(i v)$, then $B(G) \cong K_{3}$ e $K_{1, s}, s \geq 3$ and hence by Theorem 2.9, 
$\operatorname{tr}(B(G))=n^{\prime}-2$. If $G$ is the graph given in $(v)$, then $B(G) \cong B_{s, t}, s \geq 1$ and $t \geq 2$ and hence by Theorem $2.9, \operatorname{tr}(B(G))=n^{\prime}-2$.

Open Problem. If $G$ is a connected graph with $n^{\prime} \geq 3$ blocks, then chracterize $G$ for which $\operatorname{tr}(B(G))=n^{\prime}-3$.

\section{Line Graphs}

In this section, we obtain the bounds for the total resolving number of line graphs and characterize the upper bound.

Definition 4.1. The line graph $L(G)$ of a graph $G$ is a graph whose vertex set is $E(G)$ and two vertices $e$ and $f$ of $L(G)$ are adjacent if and only if they are adjacent in $G$.

Observation 4.2. For $n \geq 4, \operatorname{tr}\left(L\left(P_{n}\right)\right)=2$.

Observation 4.3. For $n \geq 3, \operatorname{tr}\left(L\left(C_{n}\right)\right)=2$.

Observation 4.4. Let $G$ be a graph of order $n$ and size $m \geq 3$. Then $2 \leq \operatorname{tr}(L(G)) \leq$ $m-1$.

Proof. Since $|V(L(G))|=m$, by Observation $2.2,2 \leq \operatorname{tr}(L(G)) \leq m-1$.

Observation 4.5. If $G ¥ P_{n}$ and $G \not C_{n}$, then $g(L(G))=3$.

Observation 4.6. $L(G)$ is a triangle free graph if and only if $G \cong P_{n}$ or $C_{n}$.

Theorem 4.7. Let $L(G)$ be a triangle free graph. Then $\operatorname{tr}(L(G))=2$.

Proof. The proof follows from Observations 4.2, 4.3 and 4.6.

Observation 4.8. $L(G)$ is unicyclic if and only if $G$ is isomorphic to either $C_{n}$ or a spider with $\Delta(G)=3$.

Theorem 4.9. If $L(G)$ is unicyclic graph, then $\operatorname{tr}(L(G))=2$.

Proof. By Observation 4.8, $G \cong C_{n}$ or a spider with $\Delta(G)=3$. If $G \cong C_{n}$, then by Observation 4.3, $\operatorname{tr}(L(G))=2$. If $G$ is a spider with $\Delta(G)=3$, then $L(G)$ is satisfies one of the condition given in Observation 2.3. By Observation 2.3, $\operatorname{tr}(L(G))=2$.

Theorem 4.10. Let $G$ be a graph of order $n$ and size $m \geq 3$. Then $\operatorname{tr}(L(G))=m-1$ if and only if $G \cong K_{3}$ or $P_{4}$ or $K_{1, n-1}$.

Proof. Assume that $\operatorname{tr}(L(G))=m-1$. By Theorem 2.5, $L(G)$ is either star or complete graph. Since $K_{1,2}$ is the line graph of $P_{4}, K_{3}$ is the line graph of $K_{1,3}$ or $K_{3}$ and $K_{m}$, $m \geq 4$ is the line graph of $K_{1, n-1}, n \geq 5, G \cong K_{3}$ or $P_{4}$ or $K_{1, n-1}$. Conversely, if $G \cong K_{3}$, then $L(G) \cong K_{3}$ and hence by Theorem $2.5, \operatorname{tr}(L(G))=m-1$. If $G \cong P_{4}$, 
then $L(G) \cong P_{3}$ and hence by Theorem 2.1, $\operatorname{tr}(L(G))=2=m-1$. If $G \cong K_{1, n-1}$, then $L(G) \cong K_{n-1}$ and hence by Theorem $2.5, \operatorname{tr}(L(G))=n-2=m-1$.

Theorem 4.11. Let $L(G)$ be a 1-connected graph. Then $\operatorname{tr}(L(G))=m-2$ if and only if $G$ is one of the following graphs.

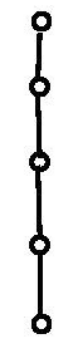

$H_{1}$

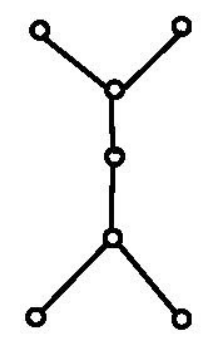

$\mathrm{H}_{2}$

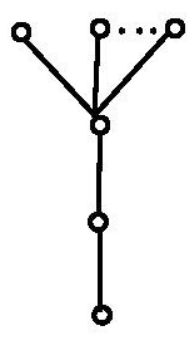

$H_{3}$

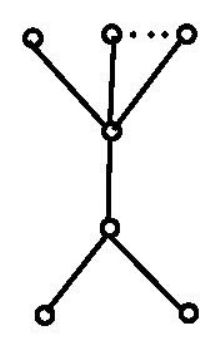

$H_{4}$

Figure 1: The graphs $G$ satisfying $\operatorname{tr}(L(G))=m-2$

Proof. Assume that $\operatorname{tr}(L(G))=m-2$. Then by Theorem 2.9, $L(G)$ is $K_{1, s} * K_{t}, s \geq 1$ and $t \geq 3$ or $K_{3} * K_{t}, t \geq 3$ or $K_{3}$ e $K_{1, s}, s \geq 3$ or $B_{s, t}(s, t \geq 1)$ or $2 K_{3}+e$. Clearly, $2 K_{3}+e$ is the line graph of $H_{2}, K_{1,1} * K_{t}, t \geq 3$ is the line graph of $B_{1, t}$ with $t \geq 2, B_{1,1}$ is the line graph of $H_{1}$ and $K_{3} * K_{t}, t \geq 3$ is the line graph of $B_{2, t}, t \geq 2$. Since $L(G)$ is $K_{1,3}$-free graph, $K_{1, s} * K_{t}, s \geq 2$ and $t \geq 3, K_{3}$ e $K_{1, s}, s \geq 3$ and $B_{s, t}, s \geq 1, t \geq 2$ are not the line graphs. Thus $G$ is one of the graphs given in Fig. 1.

Conversely, if $G \cong H_{1}$, then $L(G) \cong P_{4}$ and hence by Theorem $2.9, \operatorname{tr}(L(G))=$ $m-2$. If $G \cong H_{2}$, then $L(G) \cong 2 K_{3}+e$ and hence by Theorem $2.9, \operatorname{tr}(L(G))=$ $2=m-2$. If $G \cong H_{3}$, then $L(G) \cong K_{1,1} * K_{t}, t \geq 3$ and hence by Theorem 2.9, $\operatorname{tr}(L(G))=m-2$. If $G \cong H_{4}$, then $L(G) \cong K_{3} * K_{t}, t \geq 3$ and hence by Theorem $2.9, \operatorname{tr}(L(G))=m-2$.

Open Problem. If $L(G)$ is a 2-connected graph, then characterize $G$ for which $\operatorname{tr}(L(G))=$ $m-2$.

Open Problem. If $G$ is a connected graph of size $m \geq 3$, then characterize $G$ for which $\operatorname{tr}(L(G))=2$.

\section{Acknowledgement}

The research work of the second author is supported by the University Grants Commission, New Delhi through Basic Science Research Fellowship (vide Sanction No.F.7201/2007(BSR).

\section{References}

[1] F. Buckely, F. Harary, Distance in graphs, Addison Wesley, Reading MA, 1990. 
[2] G. Chartrand, L. Eroh, M. A. Johnson and O. R. Oellermann, Resolvability in graphs and metric dimension of a graph, Discrete Appl. Math. 105(2000), 99-113.

[3] G. Chartrand and P. Zhang, Introduction to Graph Theory, Tata McGraw Hill Education Private Limited, New Delhi (2006).

[4] Frank Harary, A Characterization of Block Graphs, Canad. Math. Bull., 6(1963), $1-6$.

[5] F. Harary and R. A. Melter, On the metric dimension of a graph, Ars Combin. 2(1976), 191-195.

[6] J. Paulraj Joseph and N. Shunmugapriya, Total Resolving Number of a Graph, Indian Journal of Mathematics, Vol 57, No. 3(2015), 323-343.

[7] J. Paulraj Joseph and N. Shunmugapriya, Total Resolving Number of Graphs - Some Characterizations, International Journal of Applied Graph Theory (Accepted) 323343.

[8] Varaporn Saenpholphat, Ping Zhang, Connected resolvability of graphs, Czechoslovak Mathematical Journal, Vol. 53, No. 4(2003), 827-840. 